\title{
Endocrine Plus - Is That the New Standard in Metastatic Breast Cancer?
}

\author{
Chair: Christian Singer \\ Participants: Rupert Bartsch ${ }^{\mathrm{b}}$ Michael Hubalek ${ }^{\mathrm{c}} \quad$ Georg Pfeiler $^{\mathrm{d}}$ \\ a Brustgesundheitszentrum Wien, Medizinische Universität Wien, Campus AKH Wien, Vienna, Austria \\ b Universitätsklinik für Innere Medizin 1, Klinische Abteilung für Onkologie, Comprehensive Cancer Center, \\ Medizinische Universität Wien, Vienna, Austria \\ ' Brustgesundheitzentrum Tirol, Univ. Frauenklinik Innsbruck, Austria \\ dOnkologische Brustambulanz, Abteilung für Gynäkologie und gynäkologische Onkologie, Medizinische Universität Wien, AKH Wien, \\ Vienna, Austria
}

\section{Question 1: Which Strategies to Combined Endocrine and Targeted Therapies in Metastatic Breast Cancer Do You See - Today and Tomorrow?}

Bartsch: When talking about the combination of endocrine treatment with targeted therapy approaches, we usually refer to the combination of everolimus plus exemestane (or tamoxifen) in metastatic breast cancer (MBC). Still, we should not forget that the combination of anti-HER2 agents plus aromatase inhibitors may be an option for highly selected patients whose tumors are co-expressing the HER2 and estrogen receptors (ER). Apart from everolimus, several other combinations of endocrine therapy with targeted agents are within different stages of clinical development (PI3K inhibitors, CDK4/6 inhibitors). Hopefully, some of those options will provide us with further chances for enhancing activity of endocrine treatment without adding relevant toxicity. In this context, the development of reliable predictive markers is pertinent, as this may ultimately enable the identification of optimal treatment sequences in individual patients.

Apart from the metastatic setting, the combination of targeted treatment approaches with endocrine therapy may become a more widely adopted treatment option in the neoadjuvant setting.

Hubalek: Strategies that combine endocrine therapy with targeted inhibitors of growth factor receptors or cell-survival pathways to further enhance first-line response have largely been disappointing, suggesting that any attempts to prevent endocrine resistance by blocking specific pathways from the outset will be futile. In contrast, success has been seen by selecting patients with acquired endocrine resistance and enhancing response to further endocrine therapy by the addition of mammalian target of rapamycin (mTOR) antagonists. Numerous other therapeutics are being evaluated in combination with endocrine therapies based on varying levels of preclinical research to support their use, including inhibitors of PI3K, HDAC, Src, IGFR-1, and CDK4/6. Enriching trial recruitment by molecular profiling of different ER+ subtypes will become increasingly important to maximize any additional benefit that these new agents may bring to current endocrine therapies for breast cancer.

Pfeiler: Endocrine treatment is highly effective with a low amount of burdensome side effects. Primary or secondary endocrine resistance might be the cause for disease progression in some cases. To overcome endocrine resistance and/or increase efficacy of this treatment modality, the addition of other 'targeted therapies' is in focus nowadays. Whereas the addition of bevacicumab to endocrine therapy failed to improve disease outcome, the mTOR inhibitor everolimus added to exemestane to overcome endocrine resistance has reached clinical routine. The ABCSG 40 trial investigates the addition of a PI3K inhibitor to endocrine treatment.

\begin{tabular}{ll}
\hline KARGER & @ 2014 S. Karger GmbH, Freiburg \\
1661-3791/14/0095-0364\$39.50/0 \\
$\begin{array}{l}\text { Fax +49 761 4520714 } \\
\text { Information@Karger.com } \\
\text { www.karger.com }\end{array}$ & $\begin{array}{l}\text { Accessible online at: } \\
\text { www.karger.com/brc }\end{array}$
\end{tabular}




\section{Question 2: Where Does 'Endocrine Plus' Fit into the Algorithm of Endocrine Treatment of Metastatic Breast Cancer?}

Bartsch: Based upon data from the BOLERO-2 and TAMRAD trials, the current place of everolimus is clearly in endocrine pretreated patients with metastatic luminal breast cancer without clear indication for chemotherapy. Due to the well-known potential side-effects, patient specific aspects such as co-morbidities, biological age and willingness to undergo more intense treatment must be taken into account.

Results from BOLERO-2 also suggested a relevant improvement in terms of progression-free survival (PFS) in firstline patients. Indeed, exemestane plus everolimus may be a first-line option in patients with more aggressive luminal disease when - based upon clinical and or biological factors - efficacy of endocrine treatment alone is doubtful. In first-line treatment of HER2-positive disease, combination of taxanes with trastuzumab plus pertuzumab is clearly the treatment of choice. In highly selected patients with low-risk HER2/ER copositive disease unwilling or unable to undergo chemotherapy, the combination of aromatase inhibitors plus anti-HER2 treatment (either trastuzumab or lapatinib) is a possible option although efficacy is clearly lower as compared to standard therapy.

Hubalek: Although the first-line MBC trials comparing a third-generation aromatase inhibitor (AI) to tamoxifen have favored the AI, one of the challenges in translating these findings into clinical practice stems from the influence of prior adjuvant endocrine therapy, particularly the increasing use of adjuvant AIs today, on the choice of endocrine agent in the advanced setting because of the development of acquired resistance. Because the majority of patients enrolled into these studies was either endocrine-treatment naïve or exposed to tamoxifen only, the 'real-life' applicability of the evidence is unclear. Because a superior dose of the selective ER downregulator fulvestrant has now been established, its role in first-line therapy is being re-established. We are now starting to see the promise realized with blocking cross-talking growth factor pathways in addition to the ER pathway. The greatest efficacy is seen with everolimus in combination with exemestane and, perhaps to a lesser extent, anti-HER2-directed therapy in combination with an AI.

Pfeiler: The combination of exemestane and everolimus is indicated in postmenopausal patients with hormone receptor positive breast cancer after treatment with ansatrozole/letrozole and recurrence or progress of the disease.

\section{Question 3: What If Endocrine Plus Fails - Are There Strategies Beyond?}

Bartsch: Currently, there is very little evidence supporting further endocrine treatment after the use of exemestane plus everolimus. The outcome from the control group of the BELLE-3 study combining fulvestrant plus the PI3K inhibitor BKM120 in patients progressing on everolimus will therefore be relevant in order to answer this question. In this context, better understanding of resistance mechanisms in individual patients is pertinent.

In my opinion, when using 'endocrine plus' in pretreated patients, usually the next step will be the switch to chemotherapy.

Hubalek: Combination drug regimens with newer cytotoxic and biologic therapies are an effective strategy in fighting tumor growth and/or progression. These combinations can facilitate the attack on multiple intercellular processes, which may result in more efficient tumor responses. These strategies may also delay or circumvent mechanisms of drug resistance by interfering with cell survival or tumor growth pathways and the cross-talk established between them. Unfortunately, mechanisms of drug resistance to both cytotoxic and biologic therapies may ultimately limit the therapeutic efficacy of any anticancer drug, especially in heavily pretreated patients who have already exhausted many of their options. Although it is hoped that tumor gene expression profiles can help us select appropriate patients for specific treatments, development of drug resistance to chemotherapy or biologic therapy remains a major limitation. To this end, numerous ongoing and future trials are investigating novel effective combinations consisting not only of chemotherapy but also of biologic agents designed to target identified cell-signaling pathways that are active in settings of disease progression. Identifying additional biomarkers and potential drug targets may lead to the development of novel biologic/chemotherapy combinations that will ultimately extend the utility of these combinations. These advances in therapy will continue to help us overcome tumor resistance and disease progression in any given patient with breast cancer.

Pfeiler: The optimal approach after treatment failure of 'endocrine plus' is in dependence of the metastatic sites, their number and - importantly - the impairment of the patient. Therefore going one step back to single endocrine treatment could be as justified as moving on to chemotherapy.

\section{Question 4: Quality of Life Versus Survival in the Metastatic Setting - What Are the Side Effects Of Combined Endocrine Therapy?}

Bartsch: Everolimus harbors a risk for relevant toxicity; here, we are leaving the field of 'conventional' endocrine treatment and patient management may even resemble that in 
chemotherapy. Obviously, this strategy necessitates shorter intervals between visits, appropriate patient education and more intense side effect management. Based upon a strict evaluation of risks and benefits, everolimus is clearly a valuable addition to breast cancer therapy. Still, it is not a drug for all patients, as preexisting conditions such as COPD, diabetes, or cachexia need to be considered.

Hubalek: The introduction of molecular targeted therapies in combination with endocrine therapy for patients with MBC has provided treatment options that are more efficacious and better tolerated than cytotoxic therapy, the previous standard of care. These advances have led to renewed efforts to define the health-related quality of life (HRQOL) impact of disease status stabilization or improvement versus that of treatmentassociated adverse events. The distinct classes of targeted agents have unique adverse events profiles related to their specific targets; therefore, treatment considerations should include the patient's pretreatment HRQOL along with the known HRQOL effects of each drug. With more second- and third-line treatment options available for patients with $\mathrm{MBC}$, HRQOL outcomes in earlier lines of therapy may guide treatment decisions for subsequent therapy, as poor HRQOL at therapy onset predicts poor survival.

Pfeiler: In the metastatic setting, quality of life is a major target of treatment. Reducing disease burden by effective combined endocrine therapy can lead to quality of life improvement. However, side effects of everolimus added to exemestane are sometimes the cause for early treatment discontinuation. Therefore treatment decision in the metastatic setting has to be made individually for each patient.

\section{Question 5: Tailoring Combined Endocrine Therapy - Who Benefits, Who Does Not?}

Bartsch: Currently, we are lacking reliable predictive markers enabling us to determine who will clearly derive benefit from everolimus (or other upcoming targeted agents complementing endocrine therapy). Given this situation, decision for using everolimus must be based upon clinical and patient specific factors. When doubts exist concerning the activity of endocrine therapy alone - e.g. tumors with low expression of hormone receptors, visceral disease without clear indication for chemotherapy, rapid progression upon prior endocrine therapy - exemestane plus everolimus is probably the treatment of choice. As outlined, patient characteristics must also factor into this algorithm.
Hubalek: Some biological features of breast cancer (Ki-67, grade, HER2/neu overexpression or amplification), which can be determined by routine immunohistochemistry analyses, can be useful in everyday practice to identify patients who are potentially unresponsive to endocrine therapy alone. Similarly, but at a higher cost, state of the art diagnostics, such as proteomics, metabolomics, microarray-based gene expression profiling and epigenetic analysis, also make patienttailored therapy increasingly possible. For example, gene expression profiling and epigenetic studies could identify potential cellular and DNA methylation biomarkers to predict and diagnose treatment related resistance. Furthermore, as drug design and genetic techniques advance, it is likely that exciting new therapies, such as agents that can restore HR positivity in HR-negative tumors, will improve the treatment and prevention of HR-positive breast cancer. These innovations will lead to the emergence of new data and more questions being answered.

Pfeiler: Even though we all know that 'one size does not fit all', we failed to determine the group of patients who will benefit - or even more important the group of patients who will not benefit from combined exemestane and everolimus. In upcoming trials some biomarkers for identification of responders early in the course of treatment or even in advance are investigated. As the combined endocrine treatment leads to some relevant side effects, predictive markers are of high interest.

\section{Participants}

PD Dr. Rupert Bartsch

Universitätsklinik für Innere Medizin 1

Klinische Abteilung für Onkologie

Comprehensive Cancer Center

Medizinische Universität Wien

Währinger Gürtel 18-20

1090 Wien, Austria

rupert.bartsch@meduniwien.ac.at

PD Dr. Hubalek Michael

Brustgesundheitzentrum Tirol

Univ. Frauenklinik Innsbruck

Anichstrasse 35

6020 Innsbruck, Austria

michael.hubalek@i-med.ac.at

Prof. Dr. Georg Pfeiler

Onkologische Brustambulanz

Abteilung für Gynäkologie und gynäkologische Onkologie

Medizinische Universität Wien

AKH Wien

Währinger Gürtel 18-20

1090 Wien, Austria

georg.pfeiler@meduniwien.ac.at 